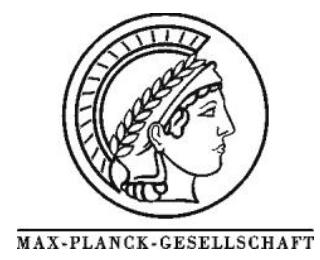

\title{
Oxidative dehydrogenation of 9,10-dihydroanthracene using multi-walled carbon nanotubes
}

\author{
D. Bégin, ${ }^{\text {a* }}$ G.s Ulrich, ${ }^{\mathrm{b}}$ J. Amadou ${ }^{\mathrm{a}}$, D. S. Su, ${ }^{\mathrm{c}}$ C. Pham-Huu, ${ }^{\mathrm{a}}$ R. Ziessel ${ }^{\mathrm{b}, *}$ \\ ${ }^{a}$ Laboratoire des Matériaux, Surfaces et Procédés pour la Catalyse (LMSPC), UMR 7515 CNRS, ECPM, \\ Université Louis Pasteur, 25, rue Becquerel, 67087 Strasbourg Cedex 02, France. \\ Part of the ELCASS (European Laboratory of Catalysis and Surface Science) \\ ${ }^{\mathrm{b}}$ Laboratoire de Chimie Organique et Spectroscopies Avancées (LCOSA), ECPM, Université Louis Pasteur, \\ 25, rue Becquerel, 67087 Strasbourg Cedex 02, France \\ c Max Planck Gesellschaft, Fritz Haber Inst, Dept Inorgan Chem, Faradayweg 4-6 D-14195 Berlin, Germany \\ Part of the ELCASS (European Laboratory of Catalysis and Surface Science)
}

" Corresponding author: e-mail ziessel@ chimie.u-strasbg.fr begind@ ecpm.u-strasbg.fr Tel: +33 39024 26 75, Fax: +33390242633

Received 16 October 2008; Received in revised form 4 December 2008; Accepted 5 December 2008; Available online 14 December 2008

\begin{abstract}
Multi-walled carbon nanotubes (MWCNTs) have been used in the oxidative dehydrogenation of 9,10-dihydroanthracene in an organic solvent (toluene) using molecular oxygen as the oxidant, and in the absence of trace amounts of metals. The process is very selective and almost no anthraquinone is formed. Thermal treatment of the MWCNTs at $2600{ }^{\circ} \mathrm{C}$ favors deoxygenation of the support and markedly improves the catalytic activity. MWCNTs are better catalysts than either charcoal or exfoliated carbon.
\end{abstract}

Keywords: Carbon nanotubes; Oxidative dehydrogenation; Anthracene; Metal free; Thermal treatment

\section{Introduction}

Carbon nanotubes (CNT) have been the subject of much research since their discovery as a by-product in arcdischarge materials in 1991 [1]. A large number of studies have been devoted to the synthesis and uses of these new materials in the field of nanotechnology and numerous potential applications in several advanced domains have been demonstrated [2-4]. Many recent publications have been focussed on the use of CNTs in supported catalysts [57]. Advantageous properties of CNTs here include their high specific surface area, and good electrical and thermal conductivity, as well as inertness towards acidic or basic reagents. In some specific cases, multi-walled carbon nanotubes (MWCNTs) have been used as supports for metallic palladium nanoparticles and as microwave absorbers [8], thereby leading to unusual chemical transformations such as regio-selective double alkynylation of substrates [8].
The present work concerns the use of MWCNTs as a catalyst for the oxidative dehydrogenation (ODH) of dihydroanthracene under mild reaction conditions and in the absence of surface loaded metals. This unusual catalytic activity is extremely interesting as it allows the avoidance of metal-based active phases which normally need to be recovered or disposed of at the end of the life of the catalyst. It also enables the use of molecular oxygen as the oxidant, which is attractive both for cost reasons and for the absence of toxic wastes. ODH reactions of 9,10dihydroanthracene have already been described in the literature [9] but none has involved the use of MWCNTs. Known procedures include the use of an oxidant in large excess [10], and/or of catalysts such as precious metals [11], strong bases [12] or Pd/charcoal [10,11]. Activated carbon has also been used as a catalyst but it has been suggested that its activity may be the result of the presence of traces of metals such as iron [13]. Herein, we describe an 
investigation of the efficacy of MWCNTs as catalysts for the dehydrogenation of 9,10-dihydroanthracene, including an assessment of the influence of surface treatment on the catalyst activity.

\section{Experimental}

\subsection{Catalyst preparation}

The multi-walled carbon nanotubes (MWCNTs) were synthesized by a catalytic route (CVD) using ethane and dihydrogen as precursors and were purified by acid and base washing as described elsewhere [14]. The yield amounted to about 2000 wt. \% with respect to the loading of the starting iron catalyst, which represents one of the highest yields cited in the literature. The very pure and homogeneous carbon nanotubes produced had a mean diameter of $c a .40 \mathrm{~nm}$ and a length of up to several hundred nanometers. The as-synthesized carbon nanotubes had both ends closed. The nanotubes were highly entangled and significant structural defects were observable on the outer wall surface. These defects were mainly due to the low synthesis temperature $\left(640^{\circ} \mathrm{C}\right)$. The as-synthesized MWCNTs were subsequently treated in flowing argon (20 $\mathrm{cm}^{3} / \mathrm{min}$ ) at $2600^{\circ} \mathrm{C}$ for $2 \mathrm{~h}$. Exfoliated graphite was provided by Carbone Lorraine (France) and activated charcoal by Calgon Carbon Corporation, Pittsburgh, USA, sample reference 9422-3793.

\subsection{Catalytic measurements}

The ODH of 9,10-dihydroanthracene (100 mg 0.555 mmol) was carried out over different carbon-based materials $(100 \mathrm{mg})$ in toluene $(10 \mathrm{~mL})$ under a molecular oxygen atmosphere $(1 \mathrm{~atm})$ at $110^{\circ} \mathrm{C}$ in a $50 \mathrm{~mL}$ round bottom flask equipped with a condenser equipped and a gas outlet. Regularly, sample of the reaction mixture (after decantation of the mixture) was analyzed after evaporation of the solvent by NMR spectroscopy. At the end of the reaction excess methanol was added to the mixture insuring complete desorption of the chemicals from the nano-tubes surface. On each case the products were isolated by column chromatography and the final products characterized by ${ }^{1} \mathrm{H}$ NMR spectroscopy in deuteriated chloroform. All reactions were performed under an atmosphere of oxygen using standard Schlenk tube techniques. All chemicals were used as received from commercial sources without further purification unless otherwise stated. Toluene was used as received and chromatographic purifications were performed using 40-63 $\mu \mathrm{m}$ silica gel. TLC was performed on silica gel plates coated with fluorescent indicator.

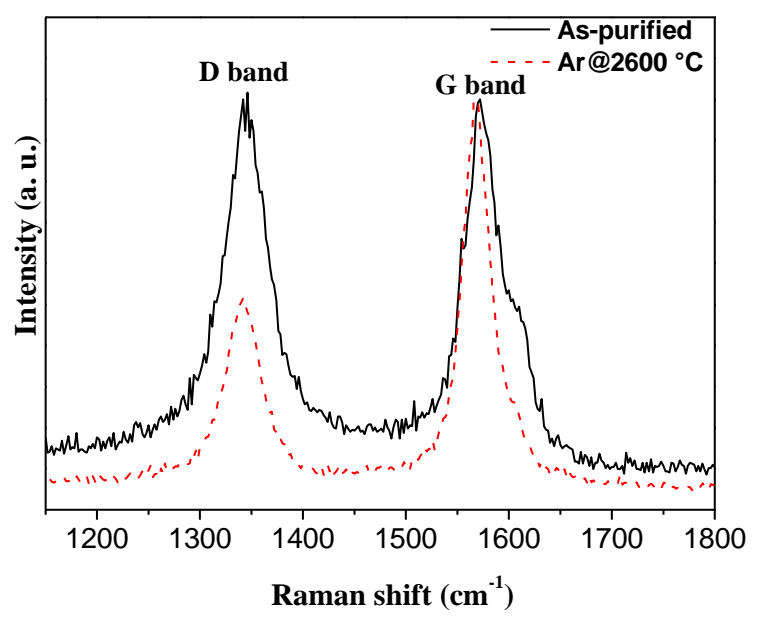

Fig. 1: Raman spectra of the MWCNTs after synthesis at $640^{\circ} \mathrm{C}$ and after heat treatment in flowing $\mathrm{Ar}$ at $2600^{\circ} \mathrm{C}$.

\subsection{NMR characterization}

${ }^{1} \mathrm{H}$ NMR (300.1 MHz) and ${ }^{13} \mathrm{C}$ NMR $(75.5 \mathrm{MHz})$ spectra were recorded at r.t. on a Bruker Advance $300 \mathrm{MHz}$ spectrometer, using perdeuteriated solvants as internal standards. FT-IR spectra were recorded as $\mathrm{KBr}$ pellets. UVVis absorption spectra were recorded on a Perkin-Elmer Lamda Uvikon 933 spectrometer in $\mathrm{CH}_{2} \mathrm{Cl}_{2}$. Fast atom bombardement ( $\mathrm{FAB}$, positive mode) mass spectra were recorded with ZAB-HF-VB analytical apparatus with $m$ nitrobenzyl alcohol ( $m$-NBA) as matrix.

\section{Results and discussion}

The as-prepared MWCNTs were heated under an inert atmosphere at $2600{ }^{\circ} \mathrm{C}$ for a short period of time. The degree of graphitization was analyzed by means of Raman spectroscopy (Figure 1) based on the use of the $G$ band (graphite $\mathrm{E}_{2 \mathrm{~g}}$ vibration) at $1580 \mathrm{~cm}^{-1}$ as a measure of the abundance of $\mathrm{sp}^{2}$-hybridised carbon atoms and the $\mathrm{D}$ band at $1350 \mathrm{~cm}^{-1}$ as a measure of the abundance of disordered carbon atoms [15]. An increase in the band intensity ratio $\mathrm{I}_{\mathrm{D}} / \mathrm{I}_{\mathrm{G}}$ after the heat treatment indicated a decrease in disordered carbon and this was consistent with TEM observations (Figure 2) showing a more ordered structure. SEM observations (not shown) indicated that the general morphology of the tubes was not modified after the heat treatment.

The specific surface area of the as-synthesized carbon nanotubes, measured by $\mathrm{N}_{2}$ adsorption at $77 \mathrm{~K}$, was $160 \mathrm{~m}^{2} \mathrm{~g}^{-1}$, which is typical of values reported in the literature [16]. Consistent with the removal of surface defects, heat treatment resulted in a small decrease of the surface area to $130 \mathrm{~m}^{2} \mathrm{~g}^{-1}$. The heat treatment also significantly diminished oxygen functionality on the outer 


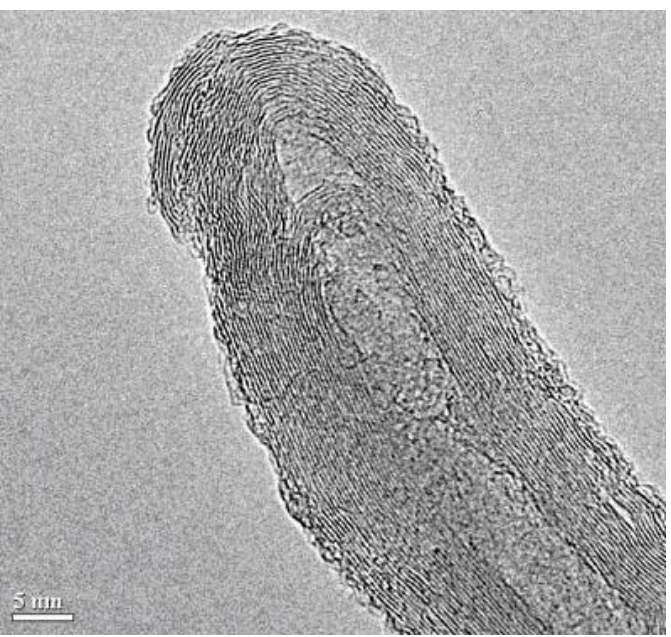

Figure 2A

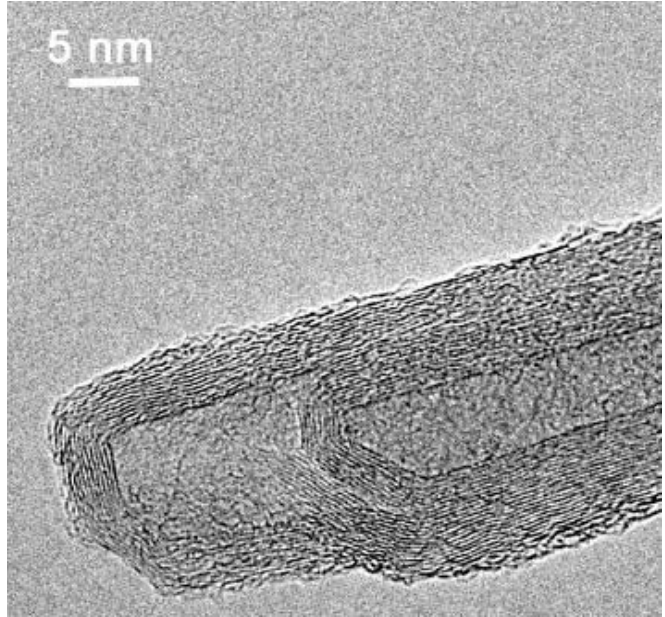

Figure 2B

Fig. 2: TEM images of MWCNTs before (2A) and after (2B) a thermal treatment at $2600^{\circ} \mathrm{C}$

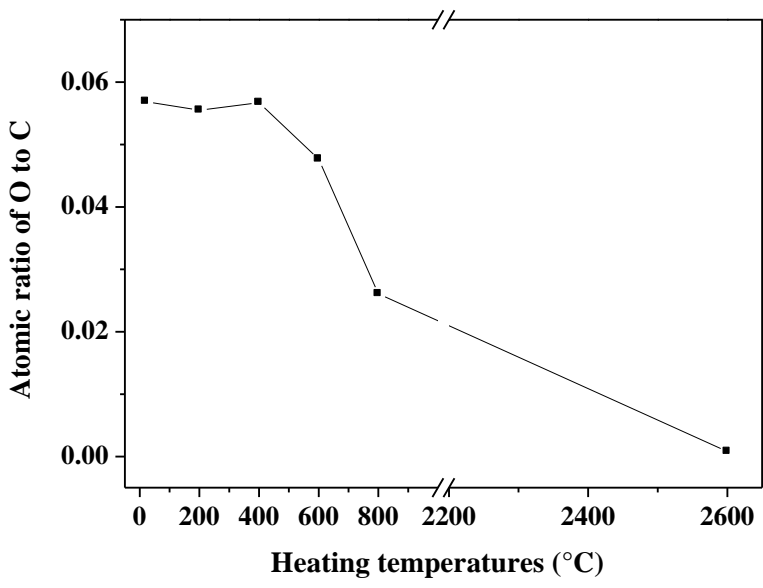

Fig. 3: Evolution of oxygen concentration at the MWCNTs surfaces as a function of temperature.

surface, as shown by XPS measurement of the surface O:C ratio (Fig ure 3). The oxygen concentration sharply decreased at $800{ }^{\circ} \mathrm{C}$ and oxygen was no longer detectable after heat treatment at $2600{ }^{\circ} \mathrm{C}$, consistent with observations by Peng et al. [17].

The ODH results are gathered in Table 1, and clearly show the chemical selectivity of the reaction no matter what catalyst was used: anthracene (A) along with only traces $(<1 \%)$ of anthraquinone $(\mathrm{Q})$ were formed.

The spectacular improvement of the ODH activity after thermal treatment of the catalyst (Figure 4), is likely attributable to the extremely low concentration of oxygenated functions on the surface as indeed shown in Figure 3. The presence of oxygenated groups on the carbon nanotubes surface could modify the strength of the adsorption of 9,10-dihydroanthracene on the surface and reduce the overall conversion rate [18]. The adsorption of aromatic

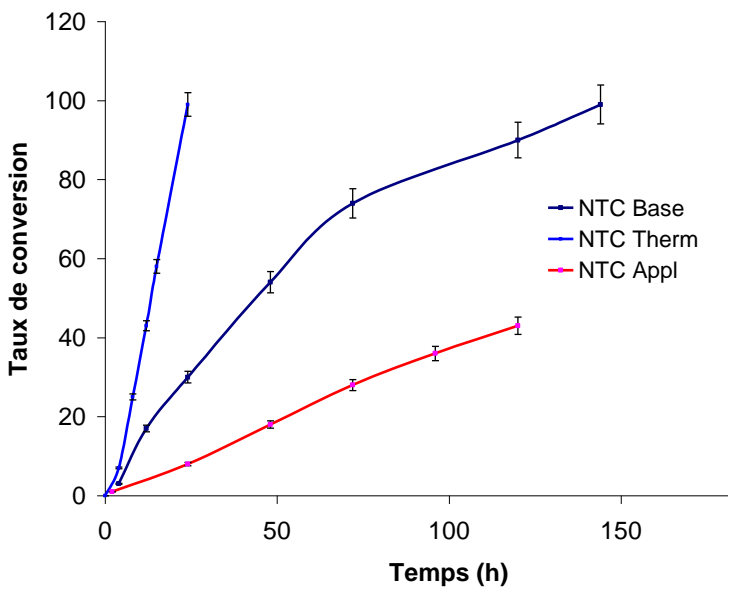

Fig. 4: Catalytic activity of various carbon based support for the synthesis of anthracene.

molecules is favoured by the delocalization and the hybridization of $\pi$-electrons between the nanotubes and the adsorbed molecules. The better catalytic activity may also reflect the better co-adsorption of oxygen, which would speed up the bimolecular surface reaction [19].

In comparing different catalyst systems, it is clear that adsorption selectivity is important and that solvent and product adsorption should be minimal. In the case of activated charcoal, the weak catalytic activity found is probably due to the marked ability of the support to adsorb the solvent and anthracene. The adsorption characteristics of the different supports was checked by UV-vis absorption at $000 \mathrm{~nm}$ on a model system based on water containing trace amounts of benzene (X mg/mL, Figure 5). As was expected, the rate of benzene absorption with activated charcoal is very fast and almost complete depletion of the benbenzene in the solution is found after $1 \mathrm{~h}$. In contrast, with 
Table 1: Catalysis results for 9,10-dihydroanthracene ODH. determined by ${ }^{1} \mathrm{H}$ NMR: $\mathrm{MWCNT}_{\text {prist }}$ as-synthesized MWCNTs without posttreatment, $\mathrm{MWCNT}_{\text {therm }}$ after heat-treatment at $2600^{\circ} \mathrm{C}$. (*) Measured by ICP-MS. (**) Reaction carried out in the absence of molecular oxygen, $\mathrm{C}_{\mathrm{ex}}=$ exfoliated graphite. $(* * *)$ from Calgon Carbon Corporation Pittburgh, USA.<smiles>c1ccc2c(c1)Cc1ccccc1C2</smiles>

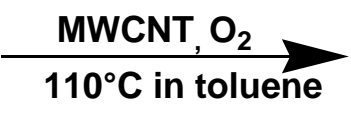<smiles>c1ccc2cc3ccccc3cc2c1</smiles>

A<smiles>O=C1c2ccccc2C(=O)c2ccccc21</smiles>

\begin{tabular}{|c|c|c|c|c|}
\hline Carbon phase & $\begin{array}{l}\text { Reaction times } \\
\text { (hours) }\end{array}$ & $\begin{array}{c}\text { Yield of A } \\
(\mathrm{mol} \%)\end{array}$ & \begin{tabular}{|c}
$\begin{array}{c}\text { Specific sur- } \\
\text { face } \\
\left(\mathbf{m}^{2} / \mathrm{g}\right)\end{array}$ \\
\end{tabular} & $\begin{array}{c}\mathrm{Fe}^{*} \\
(\mathbf{p p m})\end{array}$ \\
\hline MWCNT $_{\text {prist }}$ & $\begin{array}{c}4 \\
72 \\
144 \\
\end{array}$ & $\begin{array}{c}3 \\
74 \\
99 \\
\end{array}$ & 160 & 0.4 \\
\hline $\mathbf{M W C N T}_{\text {Therm }}$ & $\begin{array}{c}4 \\
24 \\
\end{array}$ & $\begin{array}{c}7 \\
99 \\
\end{array}$ & 130 & $<0.1$ \\
\hline MWCNT $_{\text {Therm }} * * *$ & 64 & $<1$ & 130 & - \\
\hline $\begin{array}{c}\text { Activated char- } \\
\text { coal } * * *\end{array}$ & $\begin{array}{c}4 \\
24 \\
\end{array}$ & $\begin{array}{c}4 \\
36 \\
\end{array}$ & 800 & 30 \\
\hline $\mathbf{C}_{\mathrm{Ex}}$ & 168 & 5 & 30 & $<0.1$ \\
\hline
\end{tabular}

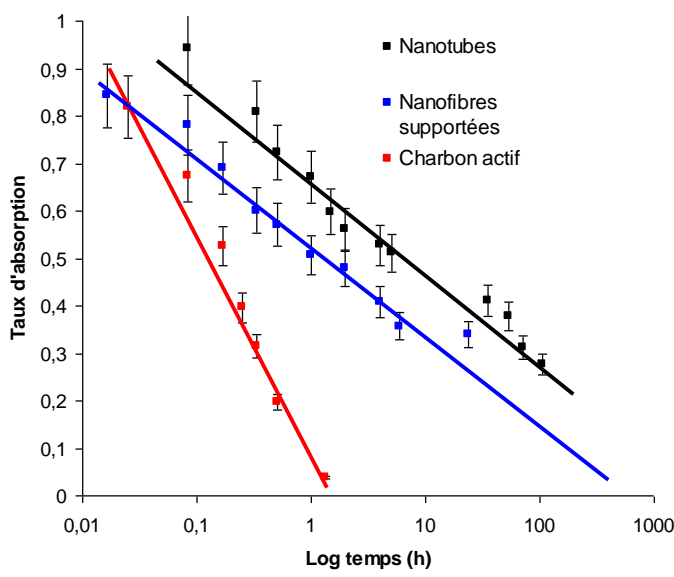

Fig. 5: Adsorption of benzene present in water as a function of contact time.

MWCNTs the adsorption process is slower by at least two orders of magnitude and complete adsorption of benzene was not attained even after $100 \mathrm{~h}$ contact (Figure 5). In the case of activated charcoal, the very efficient adsorption of benzene and by extension anthracene is probably the cause of the ineffective conversion of 9,10-dihydroanthracene to anthracene. The high adsorptivity of charcoal may be due to its known microporosity, which is not present with MWCNTs. Careful monitoring of the early stages of the reaction catalysed by MWCNTs revealed the occurrence of an induction period of a few hours which may be a reflection of the low affinity of the nanotubes for the

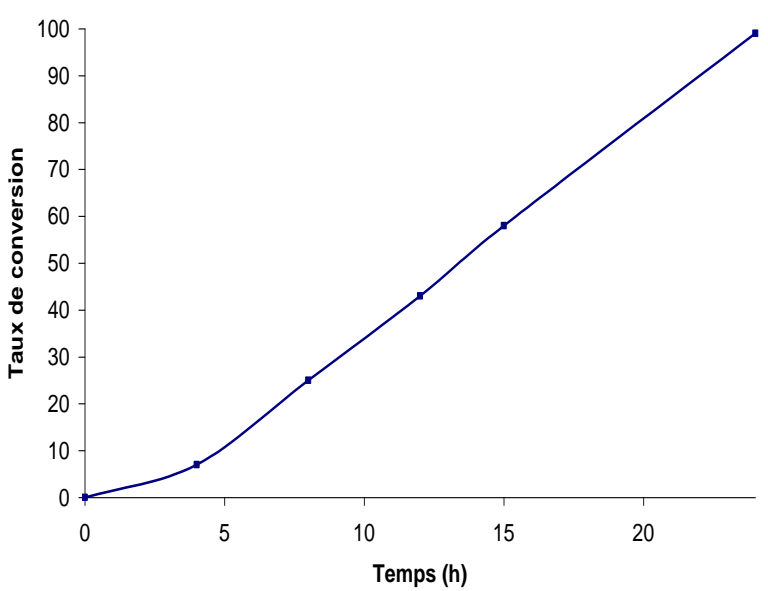

Fig. 6: Time dependence of the catalytic activity of MWCNTs Therm in the formation of anthracene

dihydroanthracene reactant and the consequent slow adsorption (Figure 6).

In the as-grown $\mathrm{MWCNT}_{\text {prist }}$ support, the carbon surface was functionalised with numerous polar, oxygencontaining groups $(-\mathrm{COOH}$ and $-\mathrm{OH})$, groups unfavourable to the chemisorption of 9,10-dihydroanthracene, which is hydrophobic. Additionally, the curvature shape of the nanotubes could play a role favourising the Van der Walls interaction of the roof shape 9,10-dihydroanthracene and consequently the catalytic reaction. Similar effects have been evoked in the literature in the case of adsorption of Krypton [23] or methane [24] on curved surfaces due to 
(a)

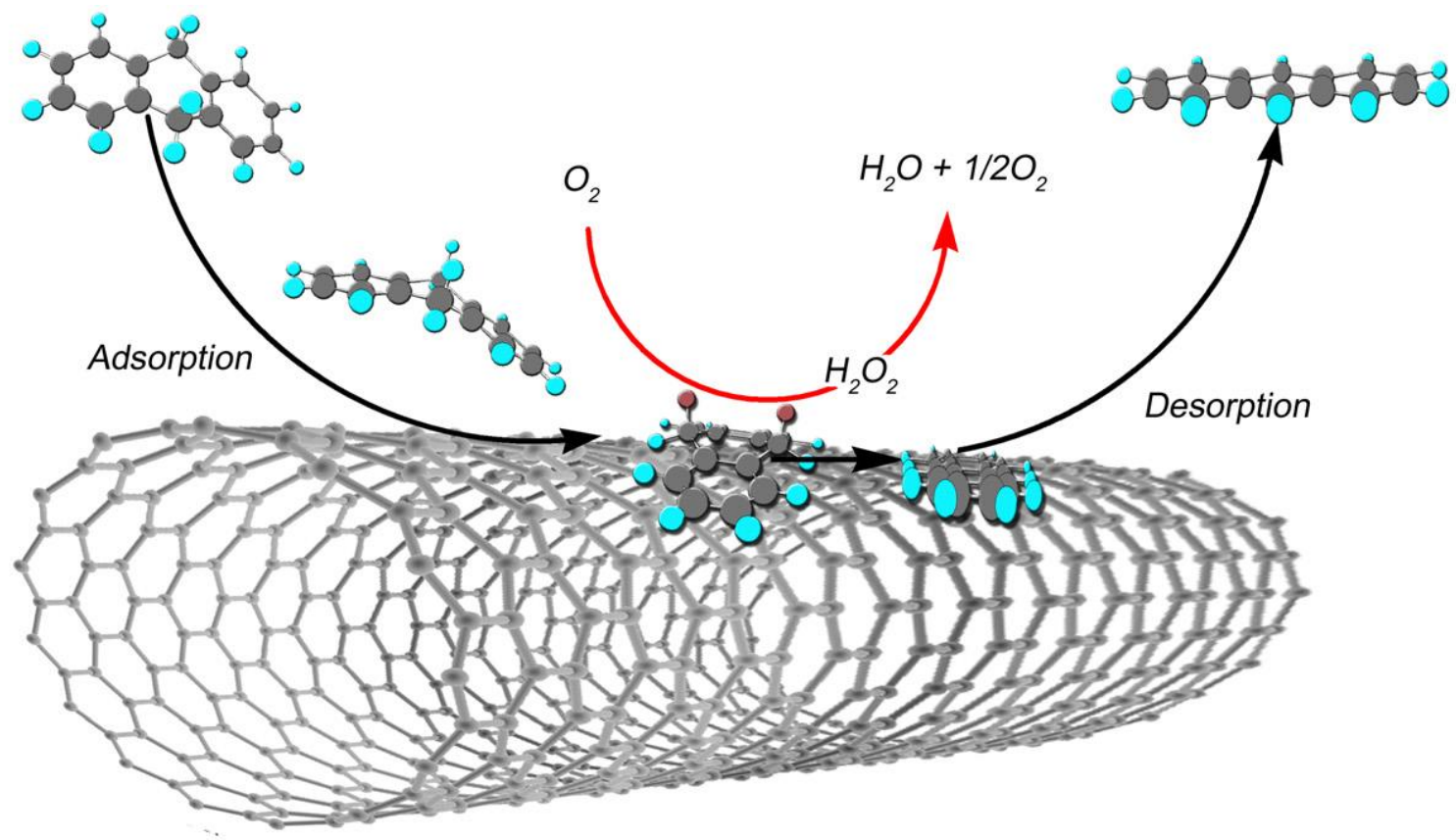

(b)
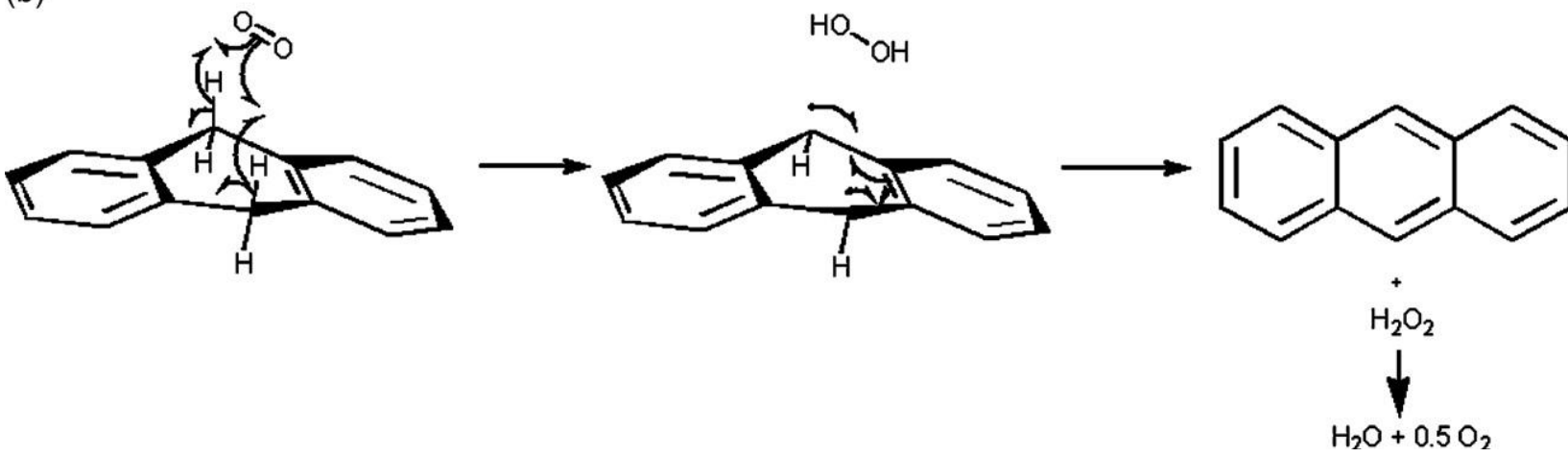

Fig. 7: (a) Top: hypothetical mechanism concerning oxidative dehydrogenation of dihydroanthracene on the surface of multi-walled carbon nanotubes. (b) Bottom: chemical drawings of electron and atom balance during the chemical transformation.

energetically favourable interactions. For the exfoliated graphite used under similar reaction conditions, the catalytic activity is negligible. Here the surface interactions with the reactants, 9,10-dihydroanthracene and molecular oxygen, are certainlyweak due to the absence of marked curvature.

From a mechanistic view point it is envisaged that the roof shaped dihydroanthracene substrate is adsorbed on the nanotube surface. During this process the folded conformation will be distorted due to strong adsorption forces and the two hydrogen atoms pointing to the outside of the surface are likely susceptible to interact with molecular oxygen to give hydrogen peroxide and anthracene as the outcome (Fig. 7a). Under the used conditions (toluene at $110{ }^{\circ} \mathrm{C}$ ) hydrogen peroxide is decomposed to oxygen and water. The flat anthracene target is then partially desorbed and the catalytic cycle is perpetuated by interactions with the starting substrate. At the end of the catalytic run, all anthracene is desorbed by treatment with a polar solvent. During the catalytic cycle, oxygen is reduced to water (pos- sibly via the formation of $\mathrm{H}_{2} \mathrm{O}_{2}$ ), and dihydroanthracene oxidized to anthracene. Likewise isolated radicals are certainly not formed owing the absence of diquinone formation during the overall process (Fig. 7b).

These results highlight the unique properties of MWCNTs as dehydrogenation catalysts working under mild conditions (atmospheric pressure, $110{ }^{\circ} \mathrm{C}$ ). It may be noted that they appear to be of similar efficacy in catalysing the ODH conversion of ethylbenzene into styrene [10] or nbutane into 2-butene [11]. It is worth noting also that less than $0.4 \mathrm{ppm}$ of $\mathrm{Fe}$ is present in solution after the first catalytic run. This is considerably less than the iron present in commercially available carbon supports [16]. The deliberate addition of $\mathrm{Fe}\left(\mathrm{ClO}_{4}\right)_{2} \cdot 6 \mathrm{H}_{2} \mathrm{O}$ to the catalytic medium had little effect on the rate of conversion of 9,10dihydroanthracene into anthracene but a significantly greater yield of anthraquinone ( $0.4 \%$ ) was obtained.

To conclude, we have demonstrated that carbon nanotubes can be effectively used as a catalyst for oxidative dehydrogenation under mild conditions with molecular 
oxygen as the hydrogen acceptor. The ODH activity was significantly enhanced by thermal treatment of the assynthesized carbon nanotubes. The treatment at $2600^{\circ} \mathrm{C}$ decreases the specific surface area and removes oxygenated groups from the surface. The heat treated MWCNTs are not only more active than their untreated precursors but also are more active than exfoliated graphite. Adsorption of the reactants (9,10-dihydroanthracene and oxygen) is certainly a key factor in the catalytic reaction and the activity of the MWCNTs appears to be a result of the relatively weak adsorption of both reactants and products incomparison to the situation with a conventional catalyst such as activated charcoal.

\section{References}

[1] S. Iijima, Nature 354 (1991) 56.

[2] P. M. Ajayan, Chem. Rev. 99 (1999) 1787.

[3] T. W. Ebbesen in Carbon Nanotubes. Preparation and Properties, Boca Raton, CRC Press (1997).

[4] K. P. de Jong, J. W. Geus, Catal. Rev.Sci. Eng. 42 (2000) 481.

[5] N. M. Rodriguez, M.S. Kim, R. T. K. Baker, J. Phys. Chem. 98 (1994) 13108.

[6] J. Garcia, H. T. Gomes, P. Serp, P. Kalck, J. L. Figueiredo, J. L. Faria, Carbon 44, (2006) 2384.

[7] J.Amadou, D. Bégin, P. Nguyen, J.P. Tessonnier,T. Dintzer, E. Vanhaecke, M.J. Ledoux, C. Pham-huu Carbon 44 (2006) 2587.

[8] J.-H. Olivier, F. Camerel, R. Ziessel, P. Retailleau, J. Amadou, C. Pham-Huu, New J. Chem. 32 (2007) 920.

[9] P. P. Fu, R. G. Harvey, Chem. Rev. 78 (1978) 317.

[10] R. G. Harvey, L. Arzadon, J. Grant, K. Urberg, J. Am. Chem. Soc. 91 (1969) 4535.

[11] J. Blum, S. Biger, Tetrahedron Lett. 11 (1970) 1825.

[12] R. G. Harvey, L. Nazareno, H. Cho, J. Am. Chem. Soc. 95 (1973) 2376.

[13] N. Nakamichi, H. Kawabata, M. Hayashi, J. Org. Chem. 68 (2003) 8272.

\section{Acknowledgement}

The authors want to thank G. Furdin (Nancy-France) for the heat treatments. This work is supported by the European Community in the frame of ELCAT and by the CNRS (Programme Materiaux Multifonctionnels à Structures Hiérarchiques et Agence Nationale pour la Recherche). We warmly thank Professor J. Harrowfield (ISIS, ULP), for his comments on the manuscript.

[14] B. Louis, G. Gulino, R; Vieira, J; Amadou, T. Dintzer, S. Galvagno, G. Centi, M.J. Ledoux , C. Pham-Huu Catal. Today 102, (2005) 23.

[15] M. S. Dresselhaus, G. Dresselhaus, R. Saitoc, A. Joriod, Physics Reports, 409 (2005) 47.

[16] A. Peigney, C.Laurent, E. Flahaut, R. R. Basca, A. Rousset, Carbon 39 (2001) 507.

[17] X. Peng, Y. Li, Z. Luan, Z. Di, H. Wang, Jia Z. Tian, Chem. Phys. Lett 376 (200) 154.

[18] A. Kuznetsiva, D.B. Mawhinney, V. Naumenko, J. T. Jr Yates, J. Liu, R. E. Smalley Chem. Phys. Lett. 321 (2000) 292.

[19] H. Ulbricht, G. Moos, T. Hertel, Phys. Rev. B 66 (2002) 075404.

[20] A. Bougrine, N. Dupont-Pavlovsky, J. Ghanbaja, D. Billaud, F. Béguin Surf. Sci. 506 (2002) 137.

[21] E.B. Mackie, R.A. Wolfson, L.M. Arnold, K. Lafdi, A.D. Migone, Langmuir 13 (1997) 7197.

[22] D.S. Su, N. Maksimova, J.J., Delgado, N. Keller, G. Mestl, M. J. T. Ledoux. Catal. Today, 102-103 (2005) 110. 